\title{
Experimente in evolutorischer Absicht
}

\begin{abstract}
Strategisches Nischenmanagement ist ein wichtiges Instrument zur Gestaltung von Transitionsstrategien. Es ist ein theoriebasierter Ansatz für die Planung, Durchführung und Bewertung von Experimenten mit neuen Technologien, die sich zur Lösung drängender gesellschaftlicher Probleme eignen, sich aber von alleine nicht am Markt durchsetzen. Am Beispiel nachhaltiger Mobilität wird erläutert, wie Strategisches Nischenmanagement Transitionsstrategien unterstützen kann.
\end{abstract}

$\mathrm{D}$ er Ansatz des Strategen nagements (SNM) wurde ausgehend von der Beobachtung entwickelt, dass viele verheissungsvolle technische Innovationen nie über das Prototypenstadium hinaus gelangen. Dies gilt insbesondere für technologische Alternativen, die hinsichtlich gesellschaftlich hoch bewerteter Güter, wie etwa der Nachhaltigkeit, potentielle Vorteile aufweisen. SNM versteht sich folglich als Ansatz, die Erfolgswahrscheinlichkeiten radikaler Innovationsprozessen zu verbessern und damit Transitionsstrategien zu unterstïtzen (1).

\section{Verquickung von Technologie und Gesellschaft}

Diese Zielsetzung bedeutet nun aber nicht, dass sich SNM im engeren Sinne als Steuerung von Innovationsprozessen versteht. Vielmehr wurde dieser Ansatz ausgehend von Erkenntnissen der jüngeren sozialwissenschaftlichen Technikforschung entwickelt, die gezeigt haben, dass Innovationen neben technologischen Aspekten substantiell von sozialen Prozessen beeinflusst werden, das heißt man spricht besser von sozio-technischen Innovationen. Sie entziehen sich damit vielfach zuverlässigen Prognosen oder kleinteiligen Steuerungsversuchen (2). Gründe dafür sind ferner der hohe Grad an Komplexität, der sich durch die Integration vieler Einzelinnovationen ergibt, die Abhängigkeit von Skalen- und Lerneffekten, welche zu einer engen Kopplung der Innovations- und Diffusionsphase führen, sowie damit verbundene Pfadabhängigkeiten und Lock-in Phänomene.

Dies führt dazu, dass Innovationen oft über eine gewisse Zeit mit Schutzvorrichtungen gestützt werden müssen, damit sie sich weiterentwickeln dynamische Effekte stärker berïcksichtigt, als bei einem bloßen Abtesten gegebener Designs hinsichtlich Akzeptanz oder Funktionstïchtigkeit.

\section{Zentrale Mechanismen der Nischenentwicklung}

Experimente dienen dazu, die Bedingungen für eine erfolgreiche Weiterentwicklung der sozio-technischen Innovation besser zu verstehen, um mittelfristig die Schutzmechanismen abbauen zu können. Die zentralen Mechanismen, welche von Nischenmanagern beeinflusst werden können, lassen sich unter zwei Stichworten zusammenfassen: Lernen und Einbettung ins gesellschaftliche Umfeld.

Lernen stimmt in seiner einfachsten Form mit dem konventionellen Ansatz des Abtestens von Funktionsfähigkeit und Akzeptanz überein. Dariuber hinaus muss, in der Perspektive des SNM, berücksichtigt werden, dass sich sowohl die Technologie als auch die Nutzungsmuster über die Zeit entwickeln können. Neben dem „Lernern erster Ordnung" (Entscheidung über zuvor festgelegte Fragen) muss also der Fokus auch auf „Lernern zweiter Ordnung" gelegt werden. Das heißt, Grundannahmen über Systemzusammenhänge und Einsatzbedingungen sollen hinterfragt werden können, um Hinweise auf ganz neue Entwicklungspfade nicht zu übersehen.

Unter der Umfeldeinbettung wird die Einpassung der Innovation in das aktuelle infrastrukturelle, organisatorische und rechtliche Umfeld gesehen. Sehr oft scheitern neue Technologien daran, dass sie zwar den Kunden den gewünschten Nutzen liefern könnten, der Aufbau einer speziellen materiellen oder rechtlichen Infrastruktur für den Unterhalt oder den Betrieb der Geräte oder Fahrzeuge jedoch sehr kostspielig wäre. Die Abstützung solcher Projekte auf breite Innovationsnetzwerke stellt dabei einen kritischen Faktor dar, da unterschiedliche Systemkomponenten miteinander optimiert werden müssen. In der Koordinierung dieser Netzwerke spielt die Bildung und Dynamik von gesellschaftlichen Erwartungshaltungen eine wichtige Rolle, die selber wieder zu einem Treiber von Innovationsaktivitäten werden können (4).

Über die Mechanismen des Lernens und der Umfeldeinbettung können Nischen entwickelt werden: Im günstigsten Fall können sie sich zu eigentlichen Transitionen auswachsen. Das heißt, ganze sozio-technische Regimes bilden sich entsprechend den Anforderungen der neuen Systeme um. Ein Beispiel aus der Technikgeschichte ist etwa der Übergang von den Segelschiffen zu den 
Dampfschiffen in der Hochseeschifffahrt. Die meisten Neuerungen werden jedoch keine derart große Tragweite erreichen. Eine technologische Nische kann sich auch auflösen, wenn ein spezielles Anwendungsfeld gefunden wird, welches sich im Sinne einer Marktnische behaupten kann. Lernen und Umfeldeinbettung können ferner dazu führen, dass der Schutzraum zwar nicht aufgehoben aber substantiell verändert wird. Dies geschieht etwa, wenn bestimmte Designs grundlegend erneuert werden oder wenn sich das unterstützende Innovationsnetzwerk verändert. Schliesslich kann sich eine Nische auch auflösen, weil die Erwartungen auf einen mittelfristigen Markterfolg keine hinreichende Unterstuitzung mehr erhalten.

Bislang wurde SNM erst als Konzept ausformuliert. Zwar wurde auch ein Handbuch für die Praxis veröffentlicht, bis heute existiert jedoch keine im Detail getestete Methodik (5). Die praktischen Grundsätze wurden anhand verschiedener vergleichender Studien über bereits abgeschlossene Experimente, vor allem aus dem Mobilitätssektor, abgeleitet. Der Mobilitätssektor wurde bislang besonders intensiv untersucht, da sich hier die Nachhaltigkeitsproblematik sehr dringlich darstellt und da im Verlauf der letzten dreissig Jahre verschiedene, radikale sozio-technische Innovationen zur Diskussion standen.

\section{- Nischendynamik im Mobilitätssektor}

Besondere Aufmerksamkeit erhielt im Zusammenhang mit der Thematik nachhaltiger Mobilität die Elektrifizierung des Antriebsstranges von Automobilen. Diese Option wurde seit den 1970er Jahren im Gefolge der Ölkrise, aber dann verstärkt noch Ende der 1980er Jahre im Zuge der Klima- und Luftreinhaltediskussion als besonders viel versprechende Option angesehen (6). Als Folge wurde in den 1990er Jahren weltweit eine große Reihe von Prototypen batteriebetriebener Elektrofahrzeuge entwickelt. Unterstïtzt wurden diese Entwicklungen durch die Verabschiedung des Zero Emission Act in Kalifornien, der die In-Verkehrsetzung von Nullemissionsfahrzeugen für die Automobilindustrie per Ende der 1990er Jahre mit zwingenden Quoten vorschrieb.

Die in diesem Zusammenhang aufgesetzten Entwicklungsprojekte gaben auch Anlass zu einer breiten Reihe von Pilot- und Demonstrationsexperimenten. Diese reichten vom rein technikfixierten Erproben einzelner Fahrzeugkomponenten bis zur Entwicklung umfassender Mobilitätsformen inklu- sive der Nutzungsmuster und des entsprechenden institutionellen Umfeldes. So führte etwa die deutsche Automobilindustrie ein Projekt mit konventionellen Fahrzeugen durch, welche nachträglich mit Elektromotoren umgerüstet worden waren. Der Versuch fand zwischen 1992 und 1996 auf der Insel Rügen statt und sollte vor allem die technische Funktionsfähigkeit von Einzelkomponenten wie neuen Batteriekonzepten testen. Etwa gleichzeitig führte die französische Automobilindustrie in La Rochelle ein Experiment durch, welches Nutzungsmuster und Kundenerfahrungen erfasste. Einen umfassenderen Ansatz verfolgten Ende der 1990er Jahre Schweizer Behörden in Mendrisio. In der Kleinstadt mit 10.000 Einwohnern sollten innerhalb von sechs Jahren etwa acht Prozent der Fahrzeuge durch Elektrofahrzeuge ersetzt werden. Die Nutzer mussten einen namhaften Anteil der Kosten tragen und dem Lernen und der Umfeldeinbettung der neuen Mobilitätsform wurde große Bedeutung beigemessen. Einzig bezüglich der Vernetzung war das Experiment wenig erfolgreich, da die internationale Automobilindustrie nie richtig in das Projekt eingebunden werden konnte.

SNM erlaubt es, die Ergebnisse der Experimente einerseits vor dem Hintergrund einer längerfristigen Entwicklungsperspektive zu bewerten und umgekehrt diese Ergebnisse auch für die Formulierung neuer Perspektiven fruchtbar zu machen (7). Es ist zu beachten, dass die Experimente nicht zu eng gefasst werden. Enger gefasste Perspektiven führen meist dazu, dass bestätigt wird, was schon von vorneherein vermutet wurde und dass Lernen zweiter Ordnung gar nicht erst stattfinden kann.

Heute ist die Weiterentwicklung von Batteriefahrzeugen kaum mehr auf der politischen und industriellen Agenda präsent. So musste auch die ursprüngliche Vorgabe von Verkaufsanteilen bei Neuwagen in Kalifornien erst immer weiter nach hinten verschoben und schliesslich fast gänzlich aufgehoben werden. Zeigt damit nicht gerade die Geschichte der Batteriefahrzeuge, wie gering die Hebelwirkung von Experimenten zu bewerten ist und begrenzt dies nicht auch die Reichweite des SNM-Ansatzes?

\section{Ausblick}

SNM will sich nicht daran messen lassen, den Erfolg bestimmter technologischer Alternativen vorherzusagen. Genau aus der gegenteiligen Überzeugung ist der Ansatz ja entwickelt worden. Vielmehr liefert SNM eine fruchtbare Analysestruktur, um Vernetzungs- und Lernprozesse bewusster analysie- ren und gestalten zu können. Dies lässt sich auch kurz am Beispiel der weiteren Entwicklung im Bereich der Elektrifizierung des Antriebsstrangs illustrieren: Die zentralen Ergebnisse aus der Entwicklung von Batteriefahrzeugen bezüglich Fahrzeugkonzepten, Steuerungstechnologien und Nutzungsmustern konnten in den letzten Jahren im Zusammenhang mit Hybridantrieben oder Brennstoffzellenfahrzeugen fruchtbar gemacht werden. Wenn auch Batteriefahrzeuge keinen Regimewechsel herbeigefïhrt haben und keine wesentlichen Marktnischen erobern konnten, so haben sie doch die nachfolgenden technologischen Nischen massgeblich beeinflusst. In diesem Sinne kommt einer Perspektive wie dem SNM die wichtige Rolle zu, Erfahrungen zwischen unterschiedlichen Innovationsprozessen verfügbar zu machen. Genau hier liegt auch eine wichtige Vorbedingung für die erfolgreiche Gestaltung von Transitionsstrategien.

\section{Anmerkungen}

(1) Hoogma, R./ Kemp, R./ Schot, J./ Truffer, B.: Experimenting for Sustainable Transport. The approach of Strategic Niche Management. London 2002.

(2) Rip, A./ Kemp, R.: Technological Change. In: Rayner, S./ Malone L. (Hrsg.): Human Choice and Climate Change, Vol. 2 Resources and Technology, Washington D.C. 1998, S. 327-399

(3) Truffer, B.: User Led innovation processes. The development of professional carsharing by environmentally concerned citizen. In: Innovation - The European Journal of Social Science Research, Vol. 16/ 2003, S. 139-154.

(4) Konrad, K.: Prägende Erwartungen. Szenarien als Schrittmacher der Technikentwicklung. Berlin 2004. (5) Weber, M./ Hoogma, R./ Lane, B./ Schot, J.: Experimenting with Sustainable Transport Innovations. A workbook for Strategic Niche Management. IPTS/ Twente University, Sevilla/ Enschede 1999.

(6) Sperling, D.: Future drive. Electric vehicles and sustainable transportation. Washington D.C. 1995.

(7) Truffer, B./ Metzner, A./ Hoogma, R.: The Coupling of Viewing and Doing. Strategic Niche Management and the electrification of individual transport. In: Greener Management International, Issue 37/ 2003, S. 111-124.

\section{Der Autor}

Dr. Bernhard Truffer ist Leiter des Centre for Innovation Research in the Utility Sector (CIRUS) der Schweizer Forschungseinrichtung EAWAG. Kontakt: EAWAG, 6047 Kastanienbaum, Schweiz. E-Mail: bernhard.truffer@eawag.ch 
(c) 20I0 Authors; licensee IÖW and oekom verlag. This is an article distributed under the terms of the Creative Commons Attribution Non-Commercial No Derivates License (http://creativecommons.org/licenses/by-nc-nd/3.o/), which permits unrestricted use, distribution, and reproduction in any medium, provided the original work is properly cited. 